\title{
Perfil clínico-epidemiológico e fatores associados às hepatites virais em Rondonópolis, MT
}

\section{Clinical-epidemiological profile and factors associated with viral hepatitis in Rondonópolis, MT}

\author{
Perfil clínico-epidemiológico y factores asociados a hepatitis \\ viral en Rondonópolis, MT
}

Débora Aparecida da Silva Santos ${ }^{1}$ Letícia Silveira Goulart ${ }^{2}$ Jaynne Soares de Oliveira ${ }^{3}$ Vitória Carolina Ferreira Benevenuto ${ }^{4}$

${ }^{1}$ Doutora em Recursos Naturais pela Universidade Federal de Campina Grande (UFCG). Enfermeira pela Fundação Educacional de Fernandópolis (FEF). Professora, adjunta III, Curso de Enfermagem, Universidade Federal de Rondonópolis (UFR), Área de Saúde Coletiva.

Pesquisadora nas áreas de saúde coletiva, estudos epidemiológicos e saúde ambiental. E-mail: deboraassantos@hotmail.com, ORCID: http://orcid.org/0000-0003-1862-7883

${ }^{2}$ Doutora em Ciências pela Universidade Federal do Rio Grande do Sul. Farmacêutica pela Universidade Federal de Santa Maria. Professora, associada I, Curso de Enfermagem, Universidade Federal de Rondonópolis (UFR), Brasil. E-mail: Igoulart77@yahoo.com.br, ORCID: http://orcid.org/0000-0003-1452-4908

${ }^{3}$ Acadêmica do curso de Enfermagem da Universidade Federal de Rondonópolis (UFR), bolsista de iniciação científica. E-mail: jaynnesoares0603@gmail.com; ORCID: https://orcid.org/0000-0002-6652-9957

${ }^{4}$ Acadêmica do curso de Enfermagem da Universidade Federal de Rondonópolis (UFR), bolsista de iniciação científica. E-mail: vihbene.etc@gmail.com, ORCID: https://orcid.org/0000-0001-8461-4779 
Resumo: As hepatites virais são doenças de notificação compulsória, sendo consideradas agravos de saúde mundial. O objetivo deste estudo foi analisar o perfil clínico-epidemiológico e fatores associados aos casos de hepatites virais. Estudo transversal, retrospectivo e descritivo, realizado em Rondonópolis, MT, entre 2009 e 2018. A coleta de dados utilizou fonte secundária (Sistema de Informação de Agravos de Notificação). Após categorização das variáveis, a análise descritiva foi realizada pelo Software R e aplicado Teste Qui-Quadrado de Pearson. Foram notificados 840 casos de hepatites virais, apresentando perfil prevalente: sexo feminino, faixa etária de 20 a 59 anos, ensino fundamental, raça parda e residente na zona urbana; fonte mecânica de infecção por pessoa/pessoa, vírus B e forma clínica aguda. Houve significância estatística para variáveis faixa etária, escolaridade e forma clínica. Este estudo colabora para conscientizar as autoridades de saúde locais acerca da relevância desse agravo, sua magnitude e necessidade de estratégias de combate eficientes.

Palavras-chave: hepatites virais; epidemiologia; saúde pública.

Abstract: Viral hepatitis is a notifiable disease and is considered a global health problem. This study aimed to analyze the clinical-epidemiological profile and factors associated with cases of viral hepatitis. Cross-sectional, retrospective, and descriptive study, carried out in Rondonópolis, MT, from 2009 to 2018. Data collection used a secondary source (Notifiable Diseases Information System). After categorizing the variables, descriptive analysis was performed using Software R and Pearson's Chi-Square Test was applied. Eight hundred forty cases of viral hepatitis were reported, with a prevalent profile: female, aged 20 to 59 years, elementary school, mixed-race and resident in the urban area; mechanical source of infection per person/person, B virus, and acute clinical form. There was statistical significance for variables age group, education, and clinical form. This study collaborates to raise the awareness of local health authorities about the relevance of this disease, its magnitude, and the need for efficient combat strategies.

Keywords: viral hepatitis; epidemiology; public health.

Resumen: La hepatitis viral es una enfermedad de declaración obligatoria y se considera un problema de salud mundial. El objetivo de este estudio fue analizar el perfil clínicoepidemiológico y los factores asociados a los casos de hepatitis viral. Estudio transversal, retrospectivo y descriptivo, realizado en Rondonópolis, MT, de 2009 a 2018. La recolección de datos se utilizó como fuente secundaria (Sistema de Información de Enfermedades Notificables). Luego de categorizar las variables, se realizó un análisis descriptivo mediante el Software R y se aplicó la Prueba de Chi-Cuadrado de Pearson. Se notificaron 840 casos de hepatitis viral, con un perfil prevalente: femenino, de 20 a 59 años, primaria, mestizo y residente en el área urbana; fuente mecánica de infección por persona/persona, virus B y forma clínica aguda. Hubo significación estadística para las variables grupo de edad, educación y forma clínica. Este estudio colabora para concienciar a las autoridades sanitarias locales sobre la relevancia de esta enfermedad, su magnitud y necesidad de estrategias de combate eficientes.

Palabras clave: hepatitis virales; epidemiología; salud pública. 


\section{INTRODUÇÃO}

As hepatites são doenças virais silenciosas caracterizadas como agravo de saúde mundial e que causam inflamação do fígado. São classificadas em cinco tipos, sendo A, B, C, D e E; casos não tratados podem levar ao óbito. São designadas pelas siglas: vírus da hepatite $A(H A V)$, vírus da hepatite B (HBV), vírus da hepatite C (HCV), vírus da hepatite D (HDV) e vírus da hepatite $\mathrm{E}(\mathrm{HEV})$.

Estes vírus têm em comum a predileção para infectar as células hepáticas e divergem quanto às formas de transmissão e consequências clínicas oriundas da infecção. Podem manifestar-se em duas formas, aguda e crônica, cursando geralmente como assintomática e, ocasionalmente, sintomática; os sintomas aparecem em estágio avançado em condição crônica e, por possuir amplo espectro clínico, podem ocasionar uma insuficiência hepática aguda grave. Somente os vírus B, C e D têm potencial para desenvolver formas crônicas de hepatite.

Quando sintomáticas, o indivíduo infectado pode apresentar febre, cansaço, dor abdominal, náuseas, vômitos, icterícia, tontura, mal-estar, sendo estes sintomas comuns entre as hepatites. A susceptibilidade é universal, sendo que a infecção confere imunidade permanente e específica para cada tipo de vírus. O diagnóstico se dá por meio de exames laboratoriais e testes rápidos.

Quanto às formas de transmissão, as hepatites virais podem ser classificadas em dois grupos: o grupo de transmissão fecal-oral (HAV e HEV), devido a condições de saneamento básico, higiene pessoal, qualidade da água e dos alimentos; e o grupo (HBV, HCV e HDV) com diversos mecanismos de transmissão, como o parenteral, sexual, compartilhamento de objetos contaminados (agulhas, seringas, lâminas), utensílios para colocação de piercing e confecção de tatuagens e outros instrumentos usados para uso de drogas injetáveis e inaláveis, acidentes perfurocortantes, procedimentos cirúrgicos e odontológicos e hemodiálises sem as adequadas normas de biossegurança. A transmissão por via sexual é mais comum para o HBV que para o HCV. Os vírus das hepatites B, C e D têm também a via de transmissão vertical. 
Acometem a população de forma universal, variando de região para região, de acordo com os tipos de transmissão. Conforme a Organização Mundial da Saúde (OMS), mundialmente, existem 257 milhões de pessoas infectadas pelo vírus da hepatite $\mathrm{B}, 71$ milhões pelo vírus $\mathrm{C}$ e estimativa de 20 milhões com hepatite $\mathrm{E}$ (WHO, 2017).

No Brasil, de 1999 a 2018, foram confirmados 632.814 casos de hepatites virais, notificados no Sistema de Informação de Agravos de Notificação (SINAN), sendo divididos em $167.108(26,4 \%)$ casos de hepatite A, 233.027 $(36,8 \%)$ de hepatite $B, 228.695$ (36,1\%) de hepatite C e 3.984 (0,7\%) de hepatite $D$. A distribuição dos casos de hepatites na região Centro-Oeste do Brasil se dá nas proporções de 11,2\% para hepatite A, 9,1\% para hepatite B, 3,4\% para hepatite C e 3,4\% para hepatite D. No estado de Mato Grosso, os casos se subdividem nas proporções de $20,7 \%$ para hepatite $A, 38,6 \%$ para hepatite B, 20,7\% para hepatite C e 42,2\% para hepatite D (BRASIL, 2019).

É válido destacar que, entre as atribuições da Vigilância Epidemiológica, estão conhecer o comportamento epidemiológico das hepatites virais quanto ao agente etiológico, pessoa, tempo e lugar; identificar os principais fatores de risco para as hepatites virais e ampliar estratégias de imunização; detectar, prevenir e controlar os surtos de hepatites virais; reduzir a prevalência de infecção das hepatites virais e avaliar o impacto das medidas de controle.

Sendo as hepatites um grave problema de saúde global, tendo em vista o baixo controle dessas doenças e a insuficiência de estudos sobre essa patologia na região de estudo, faz-se necessária a existência de pesquisas sobre o perfil clínico e epidemiológico das hepatites, assim como os fatores associados, o que justifica o desenvolvimento da busca pelo conhecimento com o propósito de ampliar as ações de prevenção e promoção de saúde para grupos específicos. Além disso, a avaliação dos dados é importante e indispensável para compreender a situação epidemiológica e colaborar com possíveis medidas de controle.

Autores como Amaral et al. (2008); Bandeira et al. (2018); Cruz et al. (2009); Rocha e Guedes (2012); Rodrigues Neto et al. (2012); Cunha et al. (2014); Dias et al. (2014); Oliveira et al. (2014); Ferreira, Gonçalves e Gonzaga (2017); Gomes et al. (2017); Nunes et al. (2017); Santos e Morais (2018); 
Rodrigues et al. (2018); Farias, Oliveira e Luz (2019); Marques et al. (2019) e Timóteo et al. (2020) estudaram sobre o perfil clínico e epidemiológico das hepatites virais.

Neste sentido, o objetivo deste estudo é analisar o perfil clínico e epidemiológico e os fatores associados aos casos notificados de hepatites virais em Rondonópolis, MT, no período de 2009 a 2018.

\section{MATERIAIS E MÉTODOS}

Estudo transversal, retrospectivo e descritivo, de abordagem quantitativa, realizado no município de Rondonópolis, localizado no sul do estado de Mato Grosso (MT), no período de janeiro de 2009 a dezembro de 2018.

O cenário do estudo conta com uma população estimada, em 2019, de 232.491 habitantes, com área territorial de 4.686,622 km², PIB per capita de 43.175,13 reais. De acordo com o Cadastro Nacional de Estabelecimentos de Saúde (CNES), o município dispõe de diversos estabelecimentos de saúde atendidos pelo Sistema Único de Saúde (SUS), sendo eles: dois postos de saúde, 54 centros de saúde/unidade básica, três unidades de atenção à saúde indígena, cinco policlínicas, dois prontos atendimentos, um laboratório de saúde pública, dois polos de prevenção de doenças e agravos e promoção da saúde, além de centros de especialidades, como dois de atenção psicossocial e seis de apoio à saúde da família.

A coleta de dados aconteceu durante os meses de fevereiro e março de 2020, utilizando fonte secundária, através do banco de dados do Sistema de Informação de Agravos de Notificação (SINAN), com disponibilidade pública e gratuita no Departamento de Informática do Sistema Único de Saúde (DATASUS). Cabe ressaltar que as hepatites virais são doenças de notificação compulsória, logo, as unidades de saúde devem preencher as fichas de notificação, as quais são registradas neste banco de dados.

A população do estudo é composta de todos os casos notificados de hepatites virais do município e período de estudo. Foram incluídos no estudo todos os casos notificados de hepatites virais registrados no SINAN, compreendendo o período de dez anos de 2009 a 2018 e excluídos casos ignorados e/ou em branco. 
As variáveis selecionadas para estudo foram dados sociodemográficos (sexo, faixa etária, escolaridade, raça, zona de residência, gestação e região de residência) e dados clínicos (fonte mecânica de infecção, classificação etiológica, forma clínica, classificação final, HBsAg Sorologia/virologia, AntiHBclgM Sorologia/virologia, AntiHCV Sorologia/virologia) dos casos notificados.

Os dados coletados do banco do SINAN foram armazenados no Excel e exportados para o Software Tabwin. Após a categorização das variáveis, a análise foi realizada com a utilização do Software $R$, o qual calculou as estatísticas descritivas, frequências absolutas e percentuais. A disposição dos dados foi por meio de tabelas. Foi realizado o Teste Qui-Quadrado de Pearson para verificação de associações entre o número de casos de hepatites virais e as características sociodemográficas e clínicas. Todas as análises foram realizadas ao nível de significância de $5 \%$.

Os riscos para esta pesquisa foram mínimos por se tratar de um estudo com informações de registros dos bancos de dados disponíveis de domínio público. Esta pesquisa faz parte do projeto "Hepatites Virais: um estudo epidemiológico no Estado de Mato Grosso", que foi aprovada pelo Comitê de Ética em Pesquisa da Universidade Federal de Mato Grosso, Campus Universitário de Rondonópolis, CAAE: 19545619.1.0000.8088 e Parecer: 3.613.618, estando de acordo com a Resolução n. 466/2012 (BRASIL, 2012).

\section{RESULTADOS}

No período de 2009 a 2018, foram notificados 840 casos de hepatites virais em Rondonópolis, MT, sendo 2012 o ano com maior prevalência (16,78\%) e 2018 o ano com menor (6,31\%); caracterizou-se uma média anual de 84 casos (Tabela 1).

Tabela 1 - Distribuição dos casos notificados de hepatites virais por ano de notificação. Rondonópolis, MT, 2009 a 2018

\begin{tabular}{cccc}
\hline \multirow{2}{*}{ Ano de notificação } & \multicolumn{3}{c}{ Casos de hepatites virais } \\
\cline { 2 - 4 } & $\mathbf{n}$ & $\mathbf{\%}$ & Valor $\mathbf{p}^{*}$ \\
\hline 2009 & 57 & 6,78 & $>0,005$ \\
2010 & 54 & 6,43 & \\
2011 & 74 & 8,81 &
\end{tabular}




\begin{tabular}{cccc}
\hline \multirow{2}{*}{ Ano de notificação } & \multicolumn{3}{c}{ Casos de hepatites virais } \\
\cline { 2 - 4 } & $\mathbf{n}$ & \% & Valor $\mathbf{p}^{*}$ \\
\hline 2012 & 141 & 16,78 & \\
2013 & 87 & 10,36 & \\
2014 & 109 & 12,98 & \\
2015 & 106 & 12,62 & \\
2016 & 85 & 10,12 & \\
2017 & 74 & 8,81 & \\
2018 & 53 & 6,31 \\
\hline Total & 840 & 100 \\
\hline
\end{tabular}

$\left({ }^{*}\right)$ teste de qui-quadrado de aderência.

Fonte: Dados do Sistema de Informação de Agravos de Notificação (SINAN, 2020).

A Tabela 2 apresenta o perfil sociodemográfico dos usuários notificados com hepatites virais nos 10 anos de estudo, apresentando este padrão: indivíduos do sexo feminino (52,02\%), faixa etária de 20 a 59 anos (81,07\%), com ensino fundamental (51,78\%), raça parda (48,34\%), residente na zona urbana (95\%), gestantes $(20,72 \%)$ e residentes na região sul mato-grossense (99,64\%). O valor-p maior que 0,005 indica significância estatística entre o número de casos de hepatites virais e as variáveis faixa etária, escolaridade e gestantes.

Tabela 2 - Casos notificados de hepatites virais segundo variáveis sociodemográficas em Rondonópolis, MT, no período de 2009 a 2018

\begin{tabular}{|c|c|c|c|c|c|c|c|}
\hline \multirow{3}{*}{ Variáveis sociodemográficas } & \multicolumn{7}{|c|}{ Casos de hepatites virais } \\
\hline & \multicolumn{2}{|c|}{ Total } & \multicolumn{3}{|c|}{$2009-2013$} & \multicolumn{2}{|c|}{$2014-2018$} \\
\hline & $\mathbf{n}$ & $\%$ & Valor $p^{*}$ & $\mathrm{n}$ & $\%$ & $\mathbf{n}$ & $\%$ \\
\hline \multicolumn{8}{|l|}{ Sexo } \\
\hline Masculino & 403 & 47,98 & 0,0307 & 182 & 44,07 & 221 & 51,76 \\
\hline Feminino & 437 & 52,02 & & 231 & 55,93 & 206 & 48,24 \\
\hline \multicolumn{8}{|l|}{ Faixa etária (anos) } \\
\hline$<1$ a 14 & 26 & 3,1 & $>0,005$ & 17 & 4,12 & 9 & 2,11 \\
\hline 15 a 19 & 37 & 4,4 & & 31 & 7,5 & 6 & 1,4 \\
\hline 20 a 59 & 681 & 81,07 & & 328 & 79,42 & 353 & 82,67 \\
\hline 60 ou mais & 96 & 11,43 & & 37 & 8,96 & 59 & 13,82 \\
\hline
\end{tabular}




\begin{tabular}{|c|c|c|c|c|c|c|c|}
\hline \multirow{3}{*}{ Variáveis sociodemográficas } & \multicolumn{7}{|c|}{ Casos de hepatites virais } \\
\hline & \multicolumn{2}{|c|}{ Total } & \multicolumn{3}{|c|}{$2009-2013$} & \multicolumn{2}{|c|}{$2014-2018$} \\
\hline & $\mathbf{n}$ & $\%$ & Valor $p^{*}$ & n & $\%$ & $\mathbf{n}$ & $\%$ \\
\hline \multicolumn{8}{|l|}{ Escolaridade } \\
\hline Analfabeto & 17 & 2,02 & $>0,005$ & 6 & 1,45 & 11 & 2,58 \\
\hline $\begin{array}{l}\text { Ensino fundamental } \\
\text { incompleto/completo }\end{array}$ & 435 & 51,78 & & 217 & 52,54 & 218 & 51,05 \\
\hline $\begin{array}{l}\text { Ensino médio incompleto/ } \\
\text { completo }\end{array}$ & 195 & 23,22 & & 88 & 21,31 & 107 & 25,06 \\
\hline $\begin{array}{l}\text { Educação superior } \\
\text { incompleta/completa }\end{array}$ & 43 & 5,12 & & 8 & 1,94 & 35 & 8,2 \\
\hline $\begin{array}{l}\text { Não se aplica/Ignorados/ } \\
\text { branco }\end{array}$ & 150 & 17,86 & - & 94 & 22,76 & 56 & 13,11 \\
\hline \multicolumn{8}{|l|}{ Raça } \\
\hline Branca & 355 & 42,26 & 0,0043 & 191 & 46,25 & 164 & 38,41 \\
\hline Preta & 42 & 5 & & 13 & 3,15 & 29 & 6,79 \\
\hline Parda & 406 & 48,34 & & 184 & 44,55 & 222 & 51,99 \\
\hline Indígena & 8 & 0,95 & & 6 & 1,45 & 2 & 0,47 \\
\hline Ignorados/branco & 29 & 3,45 & - & 19 & 4,6 & 10 & 2,34 \\
\hline \multicolumn{8}{|l|}{ Zona de residência } \\
\hline Urbana & 798 & 95 & 0,1159 & 386 & 93,46 & 412 & 96,49 \\
\hline Rural & 37 & 4,4 & & 23 & 5,57 & 14 & 3,28 \\
\hline Ignorados/branco & 5 & 0,6 & - & 4 & 0,97 & 1 & 0,23 \\
\hline \multicolumn{8}{|l|}{ Gestante } \\
\hline Não & 174 & 20,72 & $>0,005$ & 128 & 30,99 & 46 & 10,77 \\
\hline 10 trimestre & 12 & 1,43 & & 4 & 0,97 & 8 & 1,87 \\
\hline 2o trimestre & 38 & 4,52 & & 16 & 3,87 & 22 & 5,15 \\
\hline 3o trimestre & 38 & 4,52 & & 26 & 6,3 & 12 & 2,81 \\
\hline Idade gestacional ignorada & 2 & 0,24 & - & 1 & 0,24 & 1 & 0,24 \\
\hline $\begin{array}{l}\text { Não se aplica/Ignorados/ } \\
\text { branco }\end{array}$ & 576 & 68,57 & - & 238 & 57,63 & 338 & 79,16 \\
\hline \multicolumn{8}{|l|}{ Região de saúde de residência } \\
\hline 51013 Sul mato-grossense & 837 & 99,64 & - & 410 & 99,27 & 427 & 100 \\
\hline 51002 Baixada cuiabana & 3 & 0,36 & - & 3 & 0,73 & 0 & 0 \\
\hline
\end{tabular}

${ }^{*}$ ) teste de qui-quadrado de aderência; (-) não incluído nos cálculos do teste Fonte: Dados do Sistema de Informação de Agravos de Notificação (SINAN, 2020). 
Em relação às características clínicas das hepatites virais nos dez anos de estudo, apresentam predominância: fonte mecânica de infecção por pessoa/pessoa (71,78\%), classificação etiológica pelo vírus B (60,95\%), forma clínica aguda (56,19\%), classificação final por confirmação laboratorial (99,76\%), HBsAg sorologia/virologia reagente (62,5\%), AntiHBclgM Sorologia/Virologia não reagente $(66,78 \%)$ e AntiHCV Sorologia/Virologia não reagente (50,48\%). A variável forma clínica foi associada ao número de casos de hepatites virais ao longo dos anos de estudo (Tabela 3).

Tabela 3 - Casos notificados de hepatites virais em Rondonópolis, MT, de acordo com características clínicas no período de 2009 a 2018

\begin{tabular}{|c|c|c|c|c|c|c|c|}
\hline \multirow{3}{*}{ Variáveis clínicas } & \multicolumn{7}{|c|}{ Casos de hepatites virais } \\
\hline & \multicolumn{2}{|c|}{ Total } & \multicolumn{3}{|c|}{$2009-2013$} & \multicolumn{2}{|c|}{$2014-2018$} \\
\hline & $\mathbf{n}$ & $\%$ & Valor $p$ & $\mathbf{n}$ & $\%$ & $\mathbf{n}$ & $\%$ \\
\hline \multicolumn{8}{|l|}{ Fonte mecânica de infecção } \\
\hline Sexual & 70 & 8,33 & - & 56 & 13,56 & 14 & 3,28 \\
\hline Transfusional & 6 & 0,71 & & 4 & 0,97 & 2 & 0,47 \\
\hline Uso de drogas injetáveis & 4 & 0,48 & & 1 & 0,24 & 3 & 0,71 \\
\hline Vertical & 4 & 0,48 & & 2 & 0,48 & 2 & 0,47 \\
\hline Acidente de trabalho & 3 & 0,36 & & 3 & 0,73 & 0 & 0 \\
\hline Domiciliar & 1 & 0,12 & & 0 & 0 & 1 & 0,23 \\
\hline Tratamento dentário & 4 & 0,48 & & 3 & 0,73 & 1 & 0,23 \\
\hline Pessoa/pessoa & 603 & 71,78 & & 253 & 61,26 & 350 & 81,97 \\
\hline Alimento/água & 8 & 0,95 & & 7 & 1,69 & 1 & 0,23 \\
\hline Outros & 2 & 0,24 & & 1 & 0,24 & 1 & 0,23 \\
\hline Ignorados /branco & 135 & 16,07 & - & 83 & 20,1 & 52 & 12,18 \\
\hline \multicolumn{8}{|l|}{ Classificação etiológica } \\
\hline Vírus A & 19 & 2,26 & 0,0412 & 12 & 2,91 & 7 & 1,64 \\
\hline Vírus B & 512 & 60,95 & & 256 & 61,98 & 256 & 59,95 \\
\hline Vírus C & 280 & 33,33 & & 119 & 28,81 & 161 & 37,7 \\
\hline Vírus B + C & 5 & 0,6 & & 4 & 0,97 & 1 & 0,24 \\
\hline Ignorados /branco & 24 & 2,86 & - & 22 & 5,33 & 2 & 0,47 \\
\hline \multicolumn{8}{|l|}{ Forma Clínica } \\
\hline Hepatite aguda & 472 & 56,19 & $>0,005$ & 261 & 63,2 & 211 & 49,41 \\
\hline Hepatite crônica/portador & 329 & 39,17 & & 134 & 32,44 & 195 & 45,67 \\
\hline Inconclusivo & 25 & 2,98 & - & 6 & 1,45 & 19 & 4,45 \\
\hline
\end{tabular}




\begin{tabular}{|c|c|c|c|c|c|c|c|}
\hline \multirow{3}{*}{ Variáveis clínicas } & \multicolumn{7}{|c|}{ Casos de hepatites virais } \\
\hline & \multicolumn{2}{|c|}{ Total } & \multicolumn{3}{|c|}{$2009-2013$} & \multicolumn{2}{|c|}{$2014-2018$} \\
\hline & $\mathbf{n}$ & $\%$ & Valor $p$ & $n$ & $\%$ & n & $\%$ \\
\hline Ignorados /branco & 14 & 1,66 & - & 12 & 2,91 & 2 & 0,47 \\
\hline \multicolumn{8}{|l|}{ Classificação final } \\
\hline Confirmação laboratorial & 838 & 99,76 & - & 412 & 99,76 & 426 & 99,77 \\
\hline $\begin{array}{l}\text { Confirmação } \\
\text { clínico-epidemiológica }\end{array}$ & 2 & 0,24 & & 1 & 0,24 & 1 & 0,23 \\
\hline \multicolumn{8}{|l|}{ HBsAg Sorol/virol } \\
\hline Reagente & 525 & 62,5 & 0,0102 & 274 & 66,34 & 251 & 58,78 \\
\hline Não reagente & 298 & 35,48 & & 127 & 30,75 & 171 & 40,05 \\
\hline Inconclusivo & 1 & 0,12 & & 1 & 0,24 & 0 & 0 \\
\hline Não realizado & 14 & 1,66 & & 9 & 2,18 & 5 & 1,17 \\
\hline Ignorados /branco & 2 & 0,24 & & 2 & 0,49 & 0 & 0 \\
\hline \multicolumn{8}{|l|}{ AntiHBclgM Sorol/virol } \\
\hline Reagente & 13 & 1,55 & 0,8685 & 5 & 1,21 & 8 & 1,87 \\
\hline Não reagente & 561 & 66,78 & & 251 & 60,78 & 310 & 72,6 \\
\hline Não realizado & 264 & 31,43 & & 155 & 37,53 & 109 & 25,53 \\
\hline Ignorados /branco & 2 & 0,24 & & 2 & 0,48 & 0 & 0 \\
\hline \multicolumn{8}{|l|}{ AntiHCV Sorol/virol } \\
\hline Reagente & 284 & 33,81 & 0,3666 & 124 & 30,02 & 160 & 37,47 \\
\hline Não reagente & 424 & 50,48 & & 201 & 48,67 & 223 & 52,23 \\
\hline Não realizado & 130 & 15,48 & & 86 & 20,82 & 44 & 10,3 \\
\hline Ignorados /branco & 2 & 0,23 & & 2 & 0,49 & 0 & 0 \\
\hline
\end{tabular}

$(*)$ teste de qui-quadrado de aderência; (-) não incluído nos cálculos do teste.

Fonte: Dados do Sistema de Informação de Agravos de Notificação (SINAN), 2020.

\section{DISCUSSÃO}

Neste estudo, houve alta prevalência de casos de hepatites virais ( $n=840$ ) entre os anos 2009 e 2018; assim como um estudo realizado no período de 2007 a 2017 em Teresina, PI, ( $n=805)$ (RODRIGUES et al., 2018) e outro no Estado de Minas Gerais, entre 2005 e 2014 ( $n=23.821$ ) (FERREIRA; GONÇALVES; GONZAGA, 2017). Em Rondonópolis, MT, no ano de 2018, houve a menor prevalência de casos (6,31\%), fato que pôde ser verificado neste mesmo ano, em um estudo entre 2014 e 2018, nos estados brasileiros (18\%) (TIMÓTEO et al., 2020). 
O perfil sociodemográfico dos usuários notificados com hepatites virais nos dez anos em Rondonópolis, MT, foi: pouca diferença estatística entre sexos feminino e masculino, adultos, baixa escolaridade, pardos e residentes na zona urbana.

A pequena diferença estatística dos casos de hepatites virais entre o sexo também foi constatada nos diferentes estados brasileiros, porém com predomínio do sexo masculino (56\%) (TIMÓTEO et al., 2020). O sexo masculino preponderou, ainda, em Minas Gerais, nos anos de 2010 a 2017 (70,2\%) (BANDEIRA et al. 2018); e no Estado do Ceará, entre 2010 e 2015, (58,60\%) (MARQUES et al., 2019).

A maioria dos casos notificados de hepatites virais é em adultos com baixa escolaridade e pardos neste estudo; assemelhando-se com dados de Teresina, PI, entre 2007 e 2017, adultos (64,73\%) e baixa escolaridade (42,8\%) (RODRIGUES et al., 2018); e, de Sobral, CE, adultos (58,38\%), baixa escolaridade $(45,4 \%)$ e pardos (70\%) (MARQUES et al., 2019). Cabe revelar que as hepatites acometem a população como um todo, porém alguns fatores de risco tornam a população adulta mais exposta à infeç̧ão (RODRIGUES NETO et al., 2012).

Além disso, prevaleceram neste estudo os casos que residem na área urbana (95\%) e os concentrados na região sul mato-grossense $(99,64 \%)$. Cabe ressaltar que Rondonópolis, MT, é referência de atendimento para 18 municípios desta região. A zona urbana também é onde foram notificados os maiores números de casos no Estado da Bahia, entre 2010 e 2014, (77,42\%) (GOMES et al., 2017).

Em relação a estas variáveis sociodemográficas, houve significância estatística entre o número de casos de hepatites virais e as variáveis: faixa etária, escolaridade e gestantes. Entre 2003 e 2011, no Estado do Pará, os casos de hepatites virais também foram associados à faixa etária (OLIVEIRA et al., 2014).

Em São José dos Pinhais, PR, a idade superior a 40 anos aumentou o risco de notificações dos casos para hepatite C (RODRIGUES NETO et al., 2012). Entre 2004 e 2007, no Hospital do Servidor Público Estadual, em São Paulo, SP, a faixa etária de 30 a 39 anos obteve significância estatística para as hepatites B e C (CRUZ et al., 2009). Em São Mateus, ES, a escolaridade 
foi associada a um risco maior para o diagnóstico de casos de hepatite $B$ (DIAS et al., 2014).

Os valores ignorados ou em branco referentes à variável gestante tiveram as maiores porcentagens (68,57\%) neste estudo, seguidos das mulheres atendidas que declararam não estarem gestantes (20,72\%). É relevante destacar a importância das consultas de pré-natal e a vacinação que previne diversas doenças, entre elas, a hepatite $B$. Um estudo da prevalência de hepatite B em mulheres que gestaram em Rio Branco, AC, de 2007 a 2015, destacou que somente $12,5 \%$ foram diagnosticadas no primeiro trimestre da gestação e houve diferença significativa $(p=0,034)$ na média de idade das infectadas quando comparadas às sem infecção (SANSON et al., 2018).

Neste estudo, prevaleceu, entre as características clínicas das hepatites virais, a fonte mecânica de infecção por pessoa/pessoa (71,78\%), forma clínica aguda $(56,19 \%)$ e a classificação final por confirmação laboratorial (99,76\%), distinguindo-se do estudo em Vitória da Conquista, BA, entre 2011 e 2014, em que a forma clínica crônica $(76,67 \%)$ e o mecanismo de infecção sexual $(64,45 \%)$ tiveram as maiores prevalências entre os portadores de hepatite B (SANTOS; MORAIS, 2018). Em Aracaju, SE, entre 2007 e 2010, foi prevalente a forma clínica crônica/portador (70\%) e a classificação final por confirmação laboratorial (92,2\%) (ROCHA; GUEDES, 2012); na Bahia, predominou a fonte mecânica de infecção: ignorados ou em branco (38,49\%) e classificação final por confirmação laboratorial (93,33\%) (GOMES et al., 2017).

É importante ressaltar que as hepatites virais cursam predominantemente de maneira subclínica e que, após um período de seis meses, em que há contínua replicação viral, as infecções inicialmente agudas (tipos B e C) podem evoluir para hepatites crônicas (BRASIL, 2018).

A classificação etiológica que apresentou maior prevalência foi referente ao vírus $B(60,95 \%)$, corroborando com os resultados em Aracaju, SE, vírus B (50,5\%) (ROCHA; GUEDES, 2012), e distinguindo-se dos resultados encontrados em Minas Gerais, onde preponderou o vírus C (50\%) (BANDEIRA et al., 2018). Entre 2010 e 2014, um estudo que abordou a ocorrência de hepatites virais com análise nas taxas de incidência nas unidades federativas do Brasil identificou o vírus C como principal etiologia notificada $(46,62 \%)$ (FARIAS; OLIVEIRA; LUZ, 2019). 
Somente a variável forma clínica foi associada ao número de casos de hepatites virais ao longo dos anos de estudo. Em Teresina, PI, entre 2014 e 2017, também, identificou-se associação estatística significativa para o vírus C na forma crônica da doença (RODRIGUES et al., 2019). Cruz et al. (2009) constataram significância estatística nas formas agudas, crônicas e portadores assintomáticos na comparação entre hepatite B e C, no Estado de São Paulo, entre 2004 e 2007.

A variável que indica a replicação do vírus no fígado é a $\mathrm{HBsAg}$, prevalecendo reagente $(62,5 \%)$ no município, bem como a variável Anti-HBc IgM, que indica uma infecção recente, foi reagente (1,55\%). A prevalência dessas variáveis é discrepante quantitativamente nos municípios de Goiânia, GO, entre 2010 e 2013, para HBsAg reagente (3,4\%) (CUNHA et al., 2014), e em Canaã dos Carajás, PA, entre 2012 e 2014, para Anti-HBc IgM reagente (12,1\%) (NUNES et al., 2017).

Quanto à variável Anti-HCV, prevaleceram os não reagentes (50,48\%). Em Curionópolis, PA, em janeiro de 2013, prevaleceu reagente 0,5\% entre as amostras para os anticorpos HCV (NUNES et al., 2017). Entre pessoas com hepatopatias crônicas que foram atendidas na Santa Casa do Pará, entre 1996 e 2007, constatou-se prevalência de 35,6\% dos pacientes reagentes para anti-HCV (AMARAL et al., 2008).

Cabe ressaltar que os dados foram obtidos a partir de fontes secundárias (dados de notificação do SINAN), logo é possível que possam haver falhas no processo de investigação epidemiológica, haja vista a grande quantidade de casos ignorados. Este fato limita, porém não inviabiliza a confiabilidade dos resultados.

\section{CONCLUSÃO}

O presente estudo evidenciou o perfil clínico e epidemiológico e os fatores associados aos casos notificados de hepatites virais em Rondonópolis, MT, no período de 2009 a 2018, revelando discrepância de alguns resultados ante o cenário geral dos estudos com o tema.

As variáveis fonte mecânica de infecção, classificação etiológica e forma clínica apresentaram valores distintos de estudos realizados em 
outros estados e a nível nacional. A forma clínica aguda, ainda, apresentou associação com os números de casos de hepatites virais no município, ressaltando a importância do diagnóstico precoce.

Houve variáveis com alto índice de falha de preenchimento, constatado pelo item ignorados ou em branco, que impossibilitaram a produção e realização eficaz de atividades para alcançar o público-alvo desta patologia, fazendo-se necessária uma maior instrução dos profissionais ao preencher a ficha de notificação compulsória, objetivando melhores ações de enfrentamento à doença.

As hepatites virais têm tratamento, disponibilizado de forma gratuita pelo Sistema Único de Saúde, caracterizado por alto índice de cura. Em relação às hepatites $A$ e $B$, as vacinas obrigatórias são uma eficiente forma de prevenção. O diagnóstico precoce pode retardar a evolução da doença para um quadro crônico, desenvolvimento de sintomas mais graves, sequelas e até o óbito.

Neste sentido, este estudo colabora para atentar as autoridades de saúde locais acerca da relevância desse agravo, sua magnitude e necessidade de estratégias de combate eficientes. Não havendo existência de estudos anteriores que abordassem a prevalência de hepatites virais em Rondonópolis, MT, e a partir dos resultados deste, torna-se imprescindível a realização de outros estudos para melhor averiguação da situação de saúde referente às hepatites virais neste município.

\section{REFERÊNCIAS}

AMARAL, I. S. A.; MOIA, L. J. M. P.; SOARES, M. C. P.; MIRANDA, E. C. B. M.; SALDANHA, R. S.; TAVARES, N. C. S. Prevalência dos marcadores sorológicos das hepatites virais B, C e delta em pacientes atendidos em um hospital de referência de doenças hepáticas crônicas do Pará. Revista Paraense de Medicina, Belém, v. 22, n. 4., [s.p.], out./dez. 2008.

BANDEIRA, L. L. B.; SOUZA, C. S.; MARQUES, D. R.; PERUZINI, G. A.; GUEDES, L. V.; NETO, J. D. S. Epidemiologia das hepatites virais por classificação etiológica. Revista Sociedade Brasileira de Clínica Médica, São Paulo, v. 16, n. 4, p. 227-31, out./dez. 2018.

BRASIL. Ministério da Saúde. Secretaria de Vigilância em Saúde. Departamento de Doenças de Condições Crônicas e Infecções Sexualmente Transmissíveis (DCCI). 
Boletim epidemiológico de hepatites virais. Brasília-DF, v. 50, n. 17, [s.p.], jul. 2019.

BRASIL. Portaria n. 466, de outubro de 2012. Dispõe sobre diretrizes e normas regulamentadoras de pesquisa com seres humanos. Conselho Nacional de Saúde. Diário Oficial da União, Brasília-DF, 13 de junho de 2013, Seção 1, p. 59.

BRASIL. Ministério da Saúde. DATASUS - Departamento de Informática do Sistema Único de Saúde. Brasília-DF, [s.d.]. Disponível em: http://www2.datasus.gov.br/ DATASUS/index.php?area=0203. Acesso em: 1o set. 2020.

CRUZ, C. R. B.; SHIRASSU, M. M.; MARTINS, W. P. Comparação do perfil epidemiológico das hepatites $B$ e $C$ em um serviço público de São Paulo. Arquivos de Gastroenterologia, São Paulo, v. 46, n. 3, p. 225-9, jul./set. 2009.

CUNHA, E. B.; NUNES, R. G.; SOUTO, R. C. F. Análise dos marcadores sorológicos para hepatite $B$ em pacientes atendidos em um laboratório de análises clínicas de Goiânia, Goiás. Revista EVS - Estudos, Vida e Saúde, Goiânia, v. 41, n. 3, p. p. 583-90, nov. 2014.

DIAS, J. A.; CERUTTI JÚNIOR, C.; FALQUETO, A. Fatores associados à infecção pelo vírus da hepatite B: um estudo caso-controle no município de São Mateus, Espírito Santo. Revista Epidemiologia e Serviços de Saúde, Brasília-DF, v. 23, n. 4, p. 683-90, out./dez. 2014.

FARIAS, C. S.; OLIVEIRA, R. A. D; LUZ, M. R. M. P. As hepatites virais no Brasil: uma análise a partir dos seus territórios. Revista Raega, Curitiba, v. 46, n. 1, p. 90-109, 2019.

FERREIRA, A. M.; GONÇALVES, E. GONZAGA, L. M. O. Hepatites virais: epidemiologia dos casos notificados no estado de Minas Gerais entre 2005 e 2014. Revista Unimontes Científica, Montes Claros, v. 19, n. 1, p. 70-7, 2017.

GOMES, D. J.; BARBOSA, H. L.; SANTOS, C. L. R; FRANCO, D. N; BORGES, J. C. Panorama das Hepatites virais no estado da Bahia no período de 2010 a 2014. Revista de Inovação, Tecnologia e Ciências (RITEC), [s.I.], v. 2, n. 2, p. 30-9, 2017.

MARQUES, J. V. S.; ALVES, B. M.; MARQUES, M. V. S.; PARENTE, C. C.; SOUSA, N. A.; FEIJÃO, T. M. P. Análise sociodemográfica das hepatites virais no estado do Ceará. SANARE - Revista de Políticas Públicas, Sobral, v. 18, n. 2, p. 26-33, 2019.

NUNES, H. M.; SARMENTO, V. P.; MALHEIROS, A. P.; PAIXÃO, J. F.; COSTA, O. S. G.; SOARES, M. C. P. As hepatites virais: aspectos epidemiológicos, clínicos e de prevenção em municípios da Microrregião de Parauapebas, sudeste do estado do 
Débora Aparecida da Silva SANTOS; Letícia Silveira GOULART; Jaynne Soares de OLIVEIRA;

Vitória Carolina Ferreira BENEVENUTO

Pará, Brasil. Revista Pan-Amazônica de Saúde, Ananindeua, v. 2, n. 8, p. 31-7, 2017.

OLIVEIRA, D. F. C. V.; SOUZA, C. N. P.; LIMA, E. J. S.; ARAÚJO, A. R.; ALMEIDA, S. S.; RAMOS, E. M. L. S. Fatores associados à hepatite viral no Estado do Pará. Revista da Estatística da Universidade Federal de Ouro Preto, Ouro Preto, v. 3, n. especial, p. 475-80, 2014.

ROCHA, E. A. M; GUEDES, S. A. G. Perfil epidemiológico das hepatites virais no município de Aracajú/SE, 2007 a 2011. Revista Ideias \& Inovação, Aracaju, v. 1, n. 1, p. 33-9, 2012.

RODRIGUES, L. M. C.; FURTADO, E. Z. L.; OLIVEIRA, A. K. N.; MORAIS, J. C.; RESENDE, M. T. S.; SILVA, V. R. Mapeamento epidemiológico das hepatites hospitalares. Revista Brasileira de Promoção à Saúde, Fortaleza, v. 32, n. 8714, p. 1-12, 2019.

RODRIGUES, T. O.; OLIVEIRA, R. C. M.; MORAES, A. B.; SILVA, C. M.; GUEDES, H. L.; AZEVEDO, P. S. S.; VERDE, R. M. C. L.; ARAÚJO, R. P. N.; NASCIMENTO, M. H.; OLIVEIRA, E. H. Perfil epidemiológico das Hepatites Virais no município de Teresina/ PI no período de 2007 a 2017. REAS - Revista Eletrônica Acervo Saúde, [s.I.], v. 10, n. 5, p. 2096-104, 2018.

RODRIGUES NETO, J.; CUBAS, M. R.; KUSMA, S. Z.; OLANDOSKI, M. Prevalência da hepatite viral $C$ em adultos usuários de serviço público de saúde do município de São José dos Pinhais - Paraná. Revista Brasileira de Epidemiologia, Rio de Janeiro, v. 15, n. 3, p. 627-38, 2012.

SANSON, M. C. G.; FEITOZA, H. A. C.; SARACENI, V.; KOIFMAN, R. J.; BESSA. A. R. S. Prevalence and epidemiological profile of Hepatitis $B$ in pregnant women: a population study in a Brazilian Western Amazon city from 2007 to 2015. Revista Brasileira de Saúde Materno Infantil, Recife, v. 18, n. 4, p. 711-21, out./dez. 2018.

SANTOS, A. C. S; MORAIS, M. T. M. Perfil epidemiológico e sociodemográfico dos portadores de hepatite B de um município do sudoeste baiano. Revista Saúde. Com, v. 14, n. 1, p. 1073-80, jan./mar. 2018.

TIMÓTEO, M. V. F.; ARAUJO, F. J. R.; MARTINS, K. C. P.; SILVA, H. R; SILVA NETO, G. A. S; PEREIRA, R. A. C; PAULINO, J. S.; PESSOA, G. T.; ALVINO, V. S.; COSTA, R. H. F. Perfil epidemiológico das hepatites virais no Brasil. Research, Society and Development, Vargem Grande Paulista, v. 9, n. 6, e29963231, 2020.

World Health Organization [WHO]. Department of HIV/AIDS. Global Hepatitis Report, [s.I.], 2017. 